\title{
CASIMIR, the Caltech airborne submillimeter interstellar medium investigations receiver
}

\author{
Michael L. Edgar, Alexandre Karpov, Sean Lin, David Miller, \\ Simon J. E. Radford, and Jonas Zmuidzinas \\ California Institute of Technology, MS 320-47, Pasadena, CA 91125, USA
}

Copyright 2008 Society of Photo-Optical Instrumentation Engineers.

This paper will be published in Millimeter and Submillimeter Detectors and Instrumentation, Proc. SPIE 7020, and is made available as an electronic preprint with permission of SPIE. One print or electronic copy may be made for personal use only. Systematic or multiple reproduction, distribution to multiple locations via electronic or other means, duplication of any material in this paper for a fee or for commercial purposes, or modification of the content of the paper are prohibited. 


\title{
CASIMIR, the Caltech airborne submillimeter interstellar medium investigations receiver
}

\author{
Michael L. Edgar, ${ }^{*}$ Alexandre Karpov, Sean Lin, David Miller, \\ Simon J. E. Radford, and Jonas Zmuidzinas \\ California Institute of Technology, MS 320-47, Pasadena, CA 91125, USA
}

\begin{abstract}
CASIMIR, the Caltech Airborne Submillimeter Interstellar Medium Investigations Receiver is a multiband, far infrared and submillimeter, high resolution, heterodyne spectrometer under development for SOFIA. It is a first generation, PI class instrument. CASIMIR is designed for detailed, high sensitivity observations of warm (100 K) interstellar gas both in external galaxies and Galactic sources, including molecular clouds, circumstellar envelopes, and protostellar cores. Combining the $2.5 \mathrm{~m}$ SOFIA mirror with state of the art superconducting mixers, will give CASIMIR unprecedented sensitivity. Initially, CASIMIR will have two bands, at 1000 and $1250 \mathrm{GHz}$, and a further three bands, 550, 750, 1400 $\mathrm{GHz}$, will be added soon after. Any four bands will be available on each flight. The availability of multiple bands during each flight will allow for efficient use of flight time. For example, searches for weak lines from rare species in bright sources can be carried out on the same flight with observations of abundant species in faint or distant objects.
\end{abstract}

Keywords: heterodyne, spectrometer, FIR/submm, SOFIA

\section{INTRODUCTION}

CASIMIR, the Caltech Airborne Submillimeter Interstellar Medium Investigations Receiver, is a far-infrared (FIR) and submillimeter, very high-resolution, heterodyne spectrometer. It is being developed as a first generation, Principal Investigator class instrument for the Stratospheric Observatory For Infrared Astronomy, SOFIA. ${ }^{1,2}$ Observations with CASIMIR on SOFIA are expected to begin in 2010 and the instrument should be available to guest investigators soon after. It is anticipated SOFIA will eventually achieve a flight rate of up to 160 flights per year, with a lifetime of 20 years.

During the initial flights, CASIMIR will have two bands available, 1.0 and $1.25 \mathrm{THz}$. Soon after, three additional bands will be added, giving a total frequency coverage from $500 \mathrm{GHz}$ up to $1.4 \mathrm{THz}$. Eventually, the frequency coverage may be expanded up to $2 \mathrm{THz}$. It will be capable of covering this frequency range with a resolution of $\sim 10^{6}$.

The FIR/submm is extremely important for the investigation of both the galactic and extragalactic warm $(T \sim 100 \mathrm{~K})$, interstellar medium. This material is heated by shock waves or UV radiation, phenomena that are often associated with star formation or other high energy events, e. g., supernovae or active galactic nuclei. This excited material then re-emits either as dust continuum radiation or gas line emission. CASIMIR will be able to utilize recent advances in the sensitivity of superconducting mixers to study the fundamental rotational transitions of many astronomically significant hydride molecules, which cannot be observed with ground based telescopes.

\section{FREQUENCY BANDS}

\subsection{Channels}

Table 1 shows a selection of significant spectral lines within four of the first bands available with CASIMIR. The fifth channel, i. e., $1.4 \mathrm{THz}$, would be used almost exclusively for the $\mathrm{H}_{2} \mathrm{D}^{+}$line, see Section 2.3. It is expected that initial observations will concentrate on lines from this list. Almost all of these lines are completely unobservable from the ground.

\footnotetext{
* Contact author: mick@submm.caltech.edu, Ph: (626) 395-3740, FAX: (626) 796-8806
} 


\subsection{Water}

As can be seen from Table 1, CASIMIR is particularly well suited to investigate the abundance and excitation of interstellar water, using a number of transitions of $\mathrm{H}_{2}{ }^{18} \mathrm{O}$. While oxygen is the third most abundant element, its chemistry in interstellar clouds is poorly understood, since the atmosphere is opaque to many of its key species, such as $\mathrm{O}, \mathrm{O}_{2}$, $\mathrm{H}_{2} \mathrm{O}, \mathrm{H}_{3} \mathrm{O}^{+}$and $\mathrm{OH}$. Gas phase water also has an important role in the energy balance of molecular clouds due to radiative cooling or heating through FIR/submm rotational transitions. ${ }^{3}$ Figure 1 shows the rotational energy levels for $\mathrm{H}_{2}{ }^{18} \mathrm{O}$, indicating the large number of low excitation level transitions visible to CASIMIR. Figure 2 shows results from observations of the $547 \mathrm{GHz} \mathrm{H_{2 }}{ }^{18} \mathrm{O}$ line obtained on the Kuiper Airborne Observatory (KAO) and predictions of the line intensities that will be obtained for the same source with CASIMIR.

Table 1. A list of selected significant species and lines that will be observed with 4 of the first frequency bands of the CASIMIR instrument. The atmospheric transmissions shown are for typical SOFIA operating altitudes, $\sim 40,000 \mathrm{ft}$ or $12 \mathrm{~km}$. At the Caltech Submillimeter Observatory (CSO), on the summit of Mauna Kea at $4.1 \mathrm{~km}$ altitude, of all the lines shown, only two have an atmospheric transmittance more than $0 \%$ : $\mathrm{CH}(1 \%)$ and $\mathrm{CH}_{2}(13 \%)$.

\begin{tabular}{|c|c|c|c|}
\hline $\begin{array}{l}\text { Freq. } \\
\text { Band } \\
\text { GHz }\end{array}$ & Species & $\begin{array}{l}\text { Line } \\
\text { Freq. } \\
\mathrm{GHz}\end{array}$ & $\begin{array}{c}\text { \% Atmos. } \\
\text { Trans. } \\
\text { @12 km }\end{array}$ \\
\hline $500-600$ & $\begin{array}{c}\mathrm{H}_{2}{ }^{18} \mathrm{O} \\
\mathrm{CH} \\
\mathrm{NH}_{3} \\
\mathrm{CO}\end{array}$ & $\begin{array}{c}547 \\
532,536 \\
572 \\
576\end{array}$ & $\begin{array}{c}81 \\
98,97 \\
94 \\
80\end{array}$ \\
\hline $700-800$ & $\mathrm{H}_{2}{ }^{18} \mathrm{O}$ & 745 & 82 \\
\hline $900-1050$ & $\begin{array}{c}\mathrm{H}_{3} \mathrm{O}^{+} \\
\mathrm{CH}_{2} \\
\mathrm{NH} \\
\mathrm{H}_{2}{ }^{18} \mathrm{O} \\
\mathrm{CO}\end{array}$ & $\begin{array}{c}985 \\
946 \\
975 \\
995 \\
1037\end{array}$ & $\begin{array}{l}65 \\
99 \\
96 \\
73 \\
94\end{array}$ \\
\hline $1050-1250$ & $\begin{array}{c}\mathrm{H}_{2}{ }^{18} \mathrm{O} \\
\mathrm{HF}\end{array}$ & $\begin{array}{c}1137,1181 \\
1189,1199 \\
1232\end{array}$ & $\begin{array}{c}70,75 \\
87,81 \\
30\end{array}$ \\
\hline
\end{tabular}




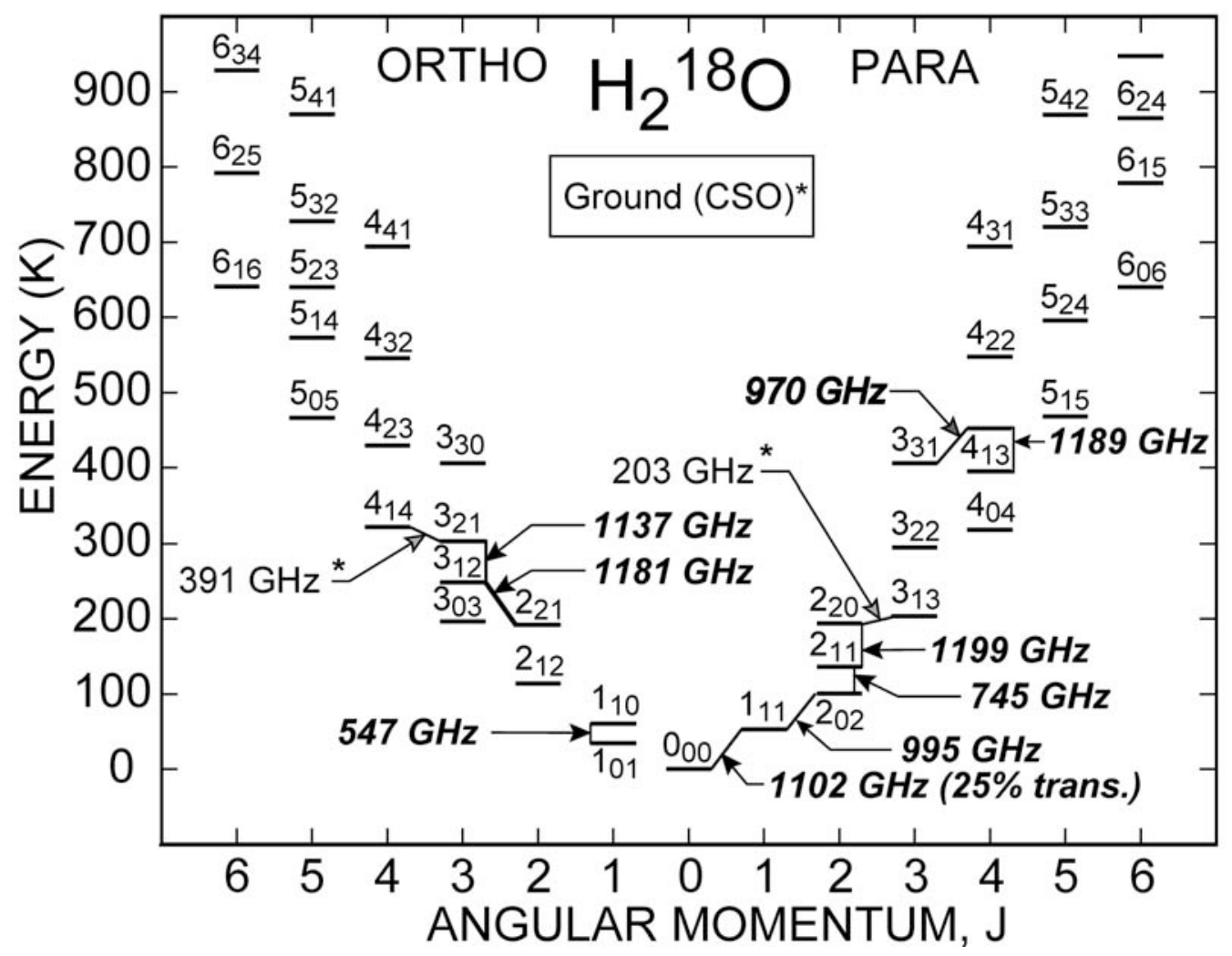

Fig. 1. CASIMIR's coverage of the rotational energy levels of the $\mathrm{H}_{2}{ }^{18} \mathrm{O}$ molecule. Four of the CASIMIR bands will be able to observe 9 transitions, including several low temperature lines, compared to only 2 relatively high energy transitions observable at the CSO, denoted by asterisks.
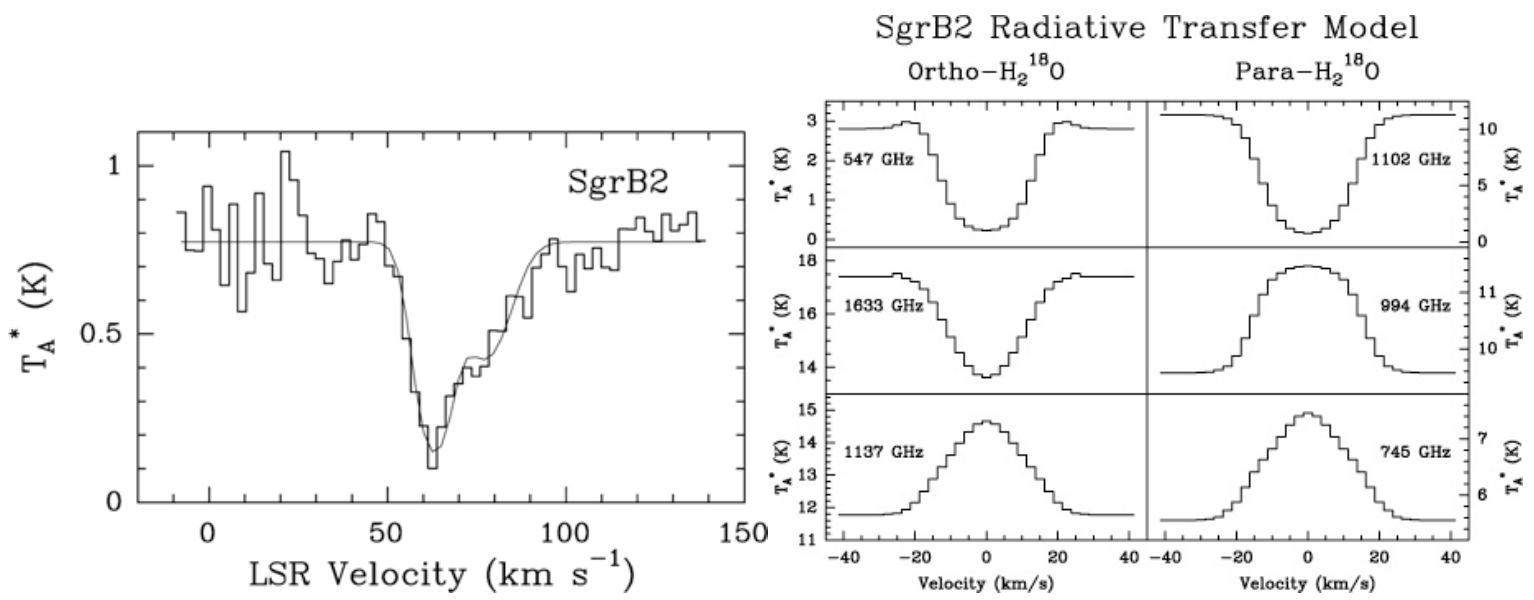

Fig. 2. Comparison of $\mathrm{H}_{2}{ }^{18} \mathrm{O}$ line sensitivities obtained with the Kuiper Airborne Observatory (KAO) and expected for CASIMIR. The left part of the figure shows $547 \mathrm{GHz}$ observations of SgrB2, obtained on the KAO. The right part shows predicted performance for observations of SgrB2 with CASIMIR on SOFIA, for several lines. SgrB2 was modeled as a sphere, $n_{\mathrm{H} 2}(r) \sim r^{-2}$ and $T(r) \sim r^{-0.5}$, which matches existing $\mathrm{CO}$, dust and $\mathrm{H}_{2}{ }^{18} \mathrm{O}$ data. 


\subsection{The $1.4 \mathrm{THz}$ band and $\mathrm{H}_{2} \mathrm{D}^{+}$}

A 1.4 THz band is expected to be available soon after CASIMIR begins observations on SOFIA. This band will concentrate on the $\mathrm{H}_{2} \mathrm{D}^{+} 1370 \mathrm{GHz}$ ground state line. The $\mathrm{H}_{2} \mathrm{D}^{+}$ion is of particular interest, since it is the deuterated version of $\mathrm{H}_{3}{ }^{+}$, which is believed to be responsible for driving much of the chemistry of molecular clouds. The $372 \mathrm{GHz}$ line for this species has now been detected from the ground in several protostellar cores with the $\mathrm{CSO}^{4}$ and with the APEX telescope ${ }^{5}$ in the Atacama Desert. However, this line traces hot, dense gas, in which the chemistry is more complicated and the abundance is expected to be low. The $1370 \mathrm{GHz}$ line is a better choice. The only previous search for this higher frequency line was made towards Orion, using the KAO, and resulted only in a tentative detection of an absorption feature. ${ }^{6}$

\section{RECEIVERS, LOCAL OSCILATORS, IF SYSTEM, AND SPECTROMETERS}

\subsection{Receivers}

The receivers for all of the first five CASIMIR bands, up to $1.4 \mathrm{THz}$, will be Nb/AlN/NbTiN Superconducting-InsulatorSuperconducting (SIS), quasi-optically coupled, twin-slot mixers. These mixers and their development are discussed in detail elsewhere. ${ }^{7}$

The ongoing development is expected to significantly reduce the noise temperatures compared to present receivers at these frequencies. It is expected that this development will achieve double side band (DSB) noise temperatures of the order of $3 h v / k_{\mathrm{B}}$ for devices operating at less than $1 \mathrm{THz}$ and $6 h v / k_{\mathrm{B}}$ above.

It is also expected that the useful frequency range of SIS mixers may be extended up to $1.6 \mathrm{THz}$.

\subsection{Local Oscillators}

The Local Oscillators (LOs) for all bands are tunerless and use solid state devices exclusively. A $1370 \mathrm{GHz}$ test LO and the flight $550 \mathrm{GHz}$ have already been acquired from Virginia Diodes. ${ }^{8}$ The $1200 \mathrm{GHz}$ flight LO was developed at JPL and is similar to the LO developed for the same frequency on the Herschel satellite. ${ }^{9}$ The $750 \mathrm{GHz} \mathrm{LO}$ will be acquired from Virginia Diodes. The $1000 \mathrm{GHz}$ and the $1.4 \mathrm{THz}$ flight LOs will be developed at JPL.

All bands are driven from a single, commercial microwave synthesizer at a frequency in the range 26-40 GHz. Any one of four bands can be selected via software at anytime during the flight, without mechanical adjustment or physical access to the instrument.

As shown in Figure 8, up to two LOs can be mounted directly to the side of the cryostat. The LO output is via a feedhorn. The output divergent beam is reflected through $90^{\circ}$ and converted into a $\sim f / 10$ converging beam, by an offaxis elliptical mirror, mounted directly below the feedhorn (see Figure 9). The beam passes through a window in the cryostat wall to a mylar beamsplitter mounted directly below the receiver elliptical mirror. The beamsplitter directs a portion of the LO signal power towards the cryostat cold work surface, combining it with the incoming, astronomical signal.

\subsection{Intermediate frequency system}

The intermediate frequency (IF) is the output signal from the mixer. For all bands on CASIMIR, the IF bandwidth is defined to be $4 \mathrm{GHz}$, centered on $6 \mathrm{GHz}$. This wide frequency range will allow observation of the broad lines from extragalactic sources.

The low noise amplifier in all bands is a Chalmers ${ }^{10}$ design. It is a two-stage amplifier using InP transistors and has a minimum gain of $27 \mathrm{~dB}$ with a nominal noise temperature of $3 \mathrm{~K}$. It is mounted on the cold work surface of the cryostat, at LHe temperature, and is connected to the mixer via a cryogenic isolator, which reduces ripple in the IF due to impedance mismatches.

The room temperature IF electronics consist of a 4-8 GHz amplifier module. This is an integrated unit developed under contract by CTT Inc., ${ }^{11}$ containing a low noise amplifier, a voltage variable attenuator (VVA), band defining filter, power amplifier, a directional coupler for monitoring the IF power level, and a switch for setting the IF power zero level. An integrated isolator at the input of the module minimizes standing wave ripples between the cryostat and amplifier module. The nominal gain of the unit is $65 \mathrm{~dB}$ with a typical noise temperature of $300 \mathrm{~K}$. The bandwidth defined by this 
unit is shown in Figure 3. A diode is connected to the monitor port for measuring the signal strength and adjusting the VVA to prevent the saturating internal amplifiers. These units are mounted directly to the side of the cryostat (see Figure 6) and are designed for fully automatic operation.

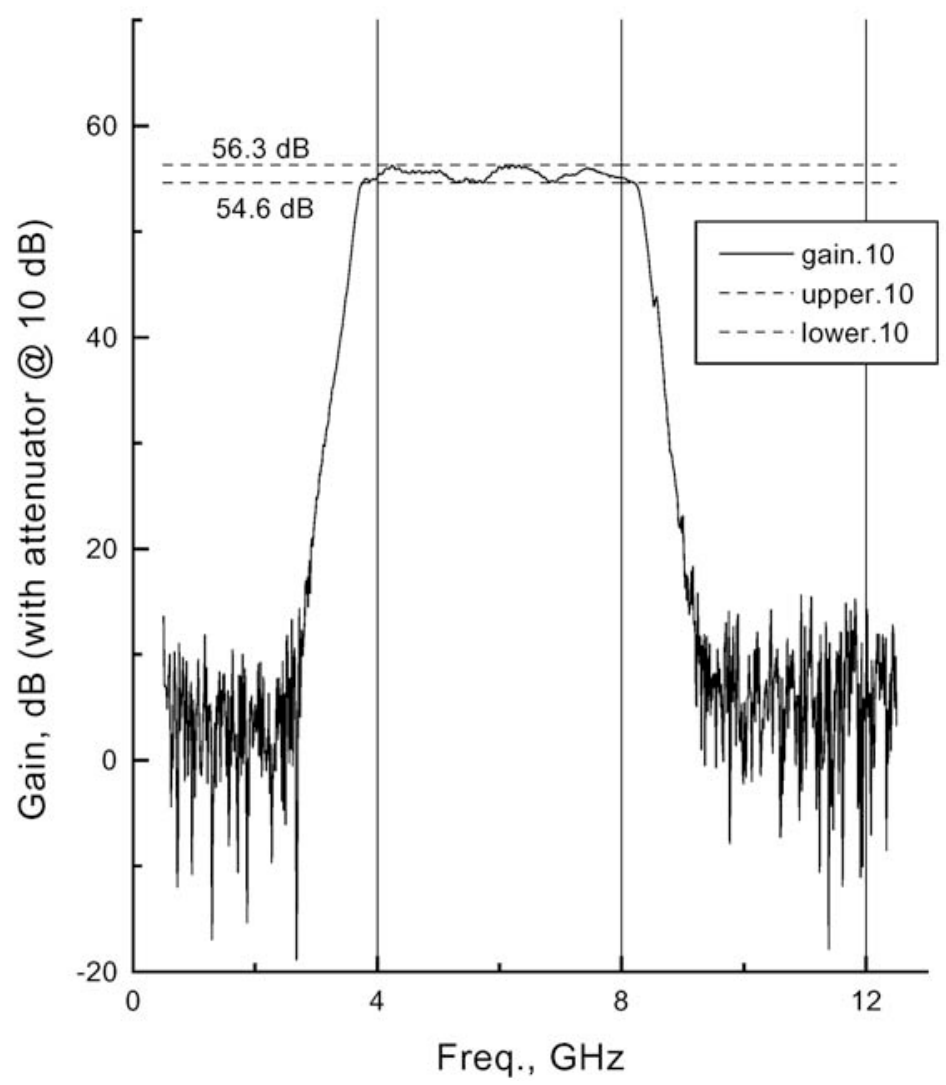

Fig. 3. IF bandwidth defined by bandpass filter within the room temperature IF unit. At a typical operating point, with the VVA set for $10 \mathrm{~dB}$ attenuation, the IF unit demonstrates excellent uniformity, $< \pm 1 \mathrm{~dB}$, across the entire $4 \mathrm{GHz}$ bandwidth.

\subsection{Microwave spectrometer}

CASIMIR will use a high resolution FFT digital spectrometer. This is a turnkey COTS design developed by Omnisys, ${ }^{12,13}$ which takes advantage of recent advances in the development of digital samplers and FPGAs. It can cover the entire $4 \mathrm{GHz}$ IF band continuously, with channel widths a small as $250 \mathrm{kHz}$, i. e., up to $16 \mathrm{k}$ channels. Observing at $1 \mathrm{THz}$, this would correspond to a resolution of $R=4 \times 10^{6}$ or $75 \mathrm{~m} \mathrm{~s}^{-1}$.

This can be achieved with only two single height, $3 \mathrm{U}$, correlator cards, with a total power consumption of less than $50 \mathrm{~W}$, which is a major advantage for an airborne instrument. The spectrometer is scalable to provide IF bandwidths of 8,12 , or $16 \mathrm{GHz}$, and we plan to be able to exploit this capability on CASIMIR in the future.

\section{INSTRUMENT CONFIGURATION AND STRUCTURE}

The general layout of the CASIMIR instrument is shown in Figure 4. Two cryostats are mounted side by side on top of a box, which contains the relay optics, see Figure 5.

Two 19-inch racks are mounted directly behind this box. All the critical electronics components are mounted in these racks, i. e., the LO drive electronics and the microwave spectrometers. This ensures very short cable runs to the cryostat and prevents any differential rotation. All electronic systems for the instrument are packaged as 19-inch bins, which will allow easy replacement of any unit. 


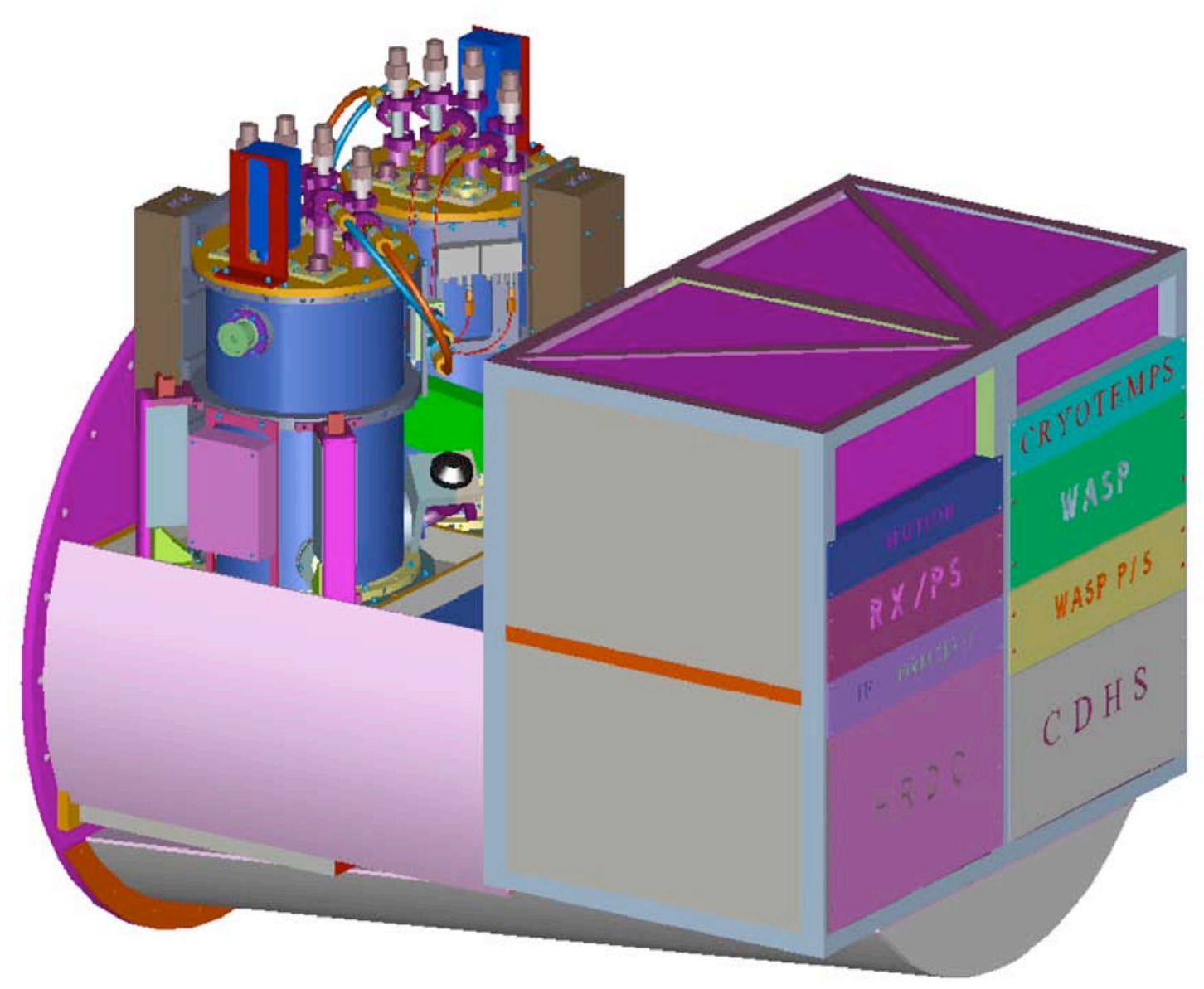

Fig. 4. The CASIMIR instrument. The instrument is mounted to the telescope via the round flange at extreme left of the figure. This flange forms the pressure interface between the telescope cavity and the aircraft's cabin. The portion of the instrument shown is located in the cabin, with the observers. The telescope beam enters the instrument through the center of this round flange. The instrument structure is constructed almost exclusively of aluminum. It is approximately $1.5 \mathrm{~m}$ long by $1 \mathrm{~m}$ square. It weighs approximately $550 \mathrm{~kg}$, including $150 \mathrm{~kg}$ of electronics mounted in the racks, at the right of the figure. Approximately $150 \mathrm{~kg}$ more of ancillary electronics are located elsewhere in the aircraft cabin. 


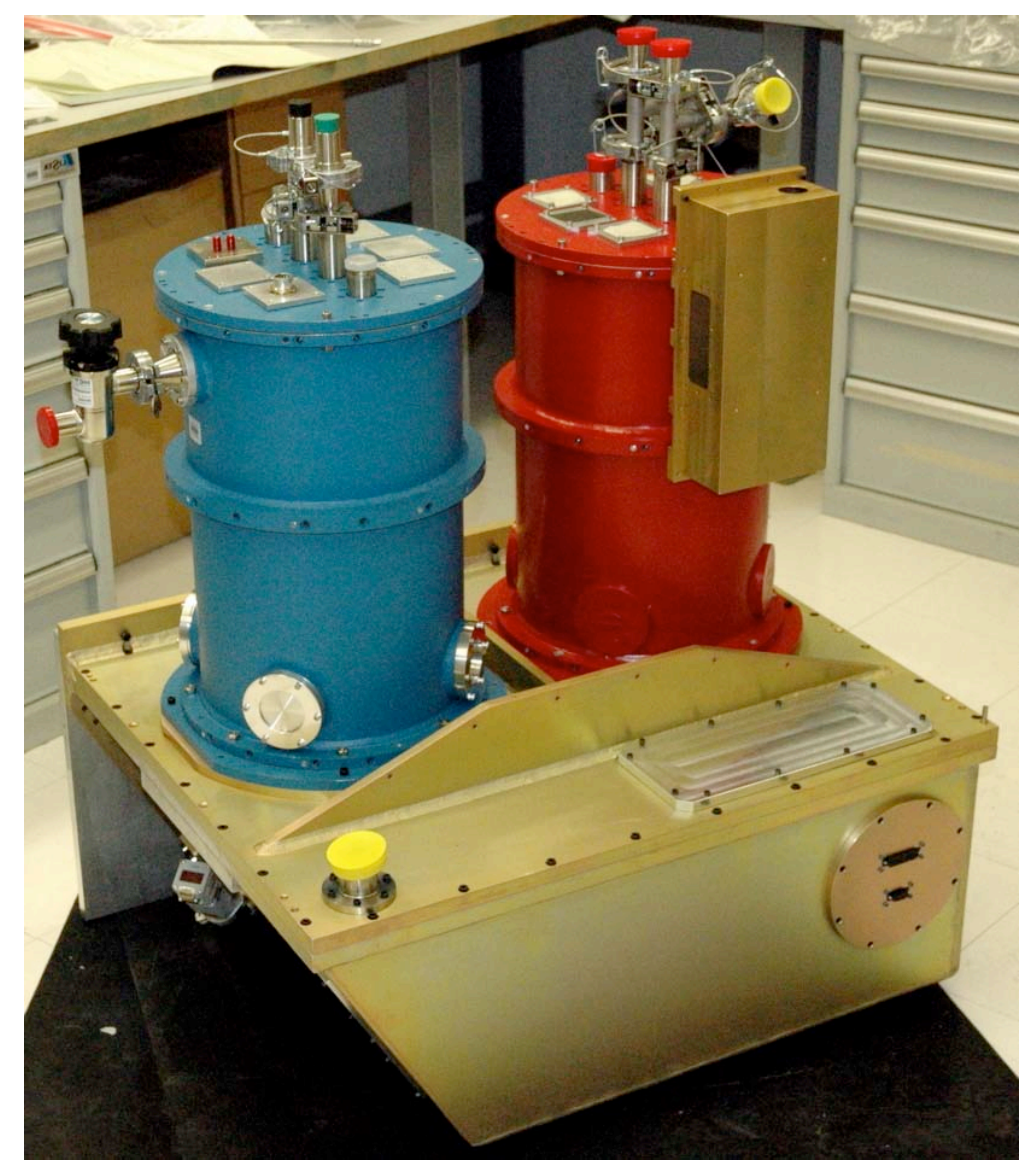

Fig. 5. The Optics Box with Cryostats Mounted. The direction of the view in this figure is approximately the same as in the previous figure. This image shows the flight hardware undergoing a pressure check. The cryostats mount directly to the top of the Optics Box. The Optics Box is constructed of welded Al 6061-T6, with dimensions of approximately $0.8 \times$ $0.7 \times 0.3 \mathrm{~m}$ and wall thicknesses varying between approximately 15 and $20 \mathrm{~mm}$. During observations, the interior of the box is exposed directly to the pressure in the telescope cavity, approximately 200 torr at $12 \mathrm{~km}$ altitude. The box is the pressure boundary between the aircraft cabin and this exterior air pressure. The baseplates of the two cryostats also form part of this pressure boundary. The telescope beam enters from the left top corner of this figure, approximately $150 \mathrm{~mm}$ below the bases of the cryostat. It is then reflected through $90 \mathrm{o}$ and directed through windows in the cryostat baseplates. This is the only pressure boundary in the entire astronomical signal beam path, i. e., between the aircraft exterior pressure and the high vacuum within the cryostat.

\subsection{Optics Box}

The Optics Box is described in general in Figure 5. Apart from providing the pressure boundary between the inside and outside of the aircraft and being the mechanical mount for the two cryostats, it also contains all of the optics that is common for all FIR/submm bands. Figure 6 shows a 3D model of the interior of the Optics Box and these optics. This can be compared with the present state of the optics within the box, shown in Figure 7.

The central feature is a plane mirror, which can be commanded to rotate through $\pm 180^{\circ}$ in the plane of the telescope and up to $\pm 5^{\circ}$ in tilt. This rotating mirror directs the telescope beam to one of the four elliptical mirrors mounted on the two cryostats, selecting the frequency band.

The calibration system consists of a chopper wheel at ambient temperature plus hot and ambient temperature loads. Moving the rotating mirror by $\sim 180^{\circ}$, allows any of the frequency bands to be first illuminated with the sky signal and then the signal from a known temperature calibration load. 


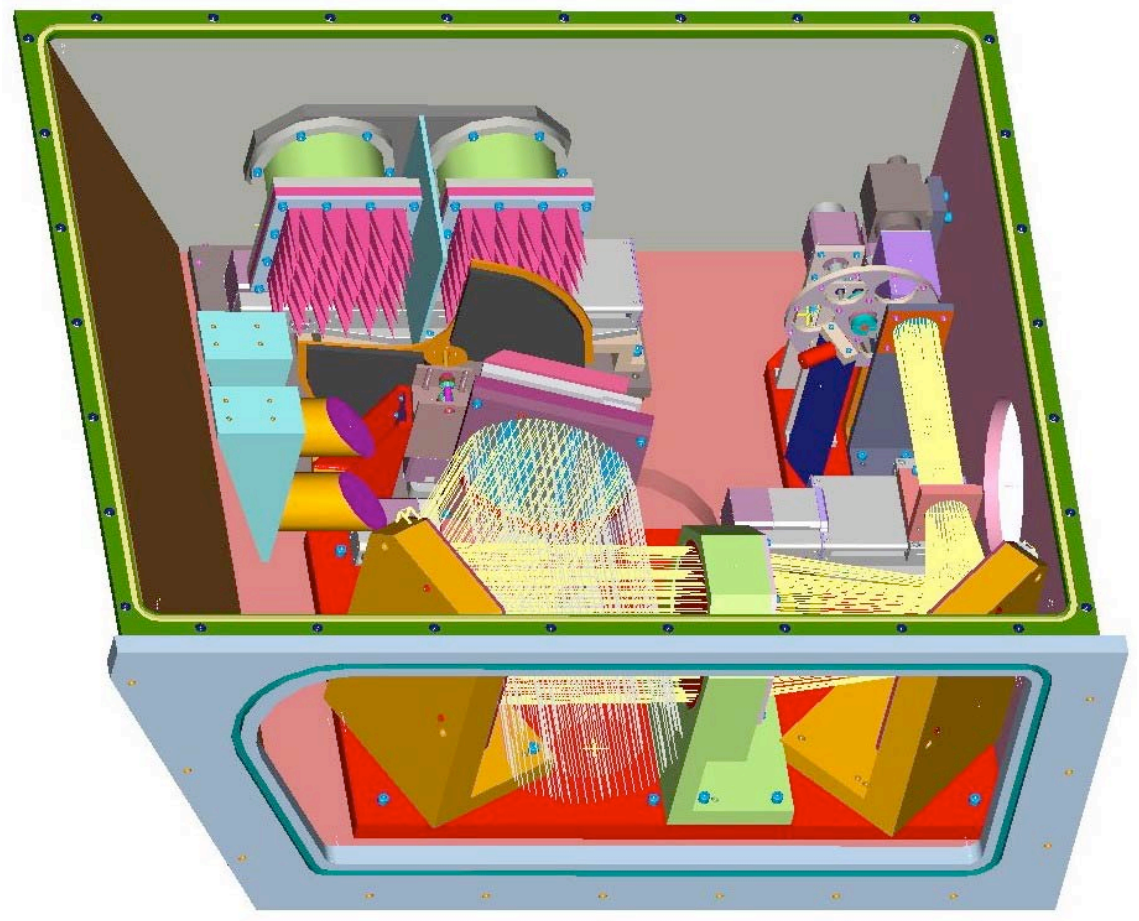

Fig. 6. The Optics Box interior. The cryostats are bolted directly to the lid of this box, which has been removed for this image. The elliptical mirrors mounted on the base of the cryostats, see Figures 8 and 9, protrude trough an aperture in the lid and are located in the plane of the telescope beam. The two elliptical mirrors for one of the cryostats are shown in the left part of the image. The telescope beam enters from the front of the figure. In this image, the rotating mirror, at the center of the figure, directs the telescope beam to the optical boresight, at the far right rear corner. The calibration chopper wheel and the two loads are shown in the rear of the figure.

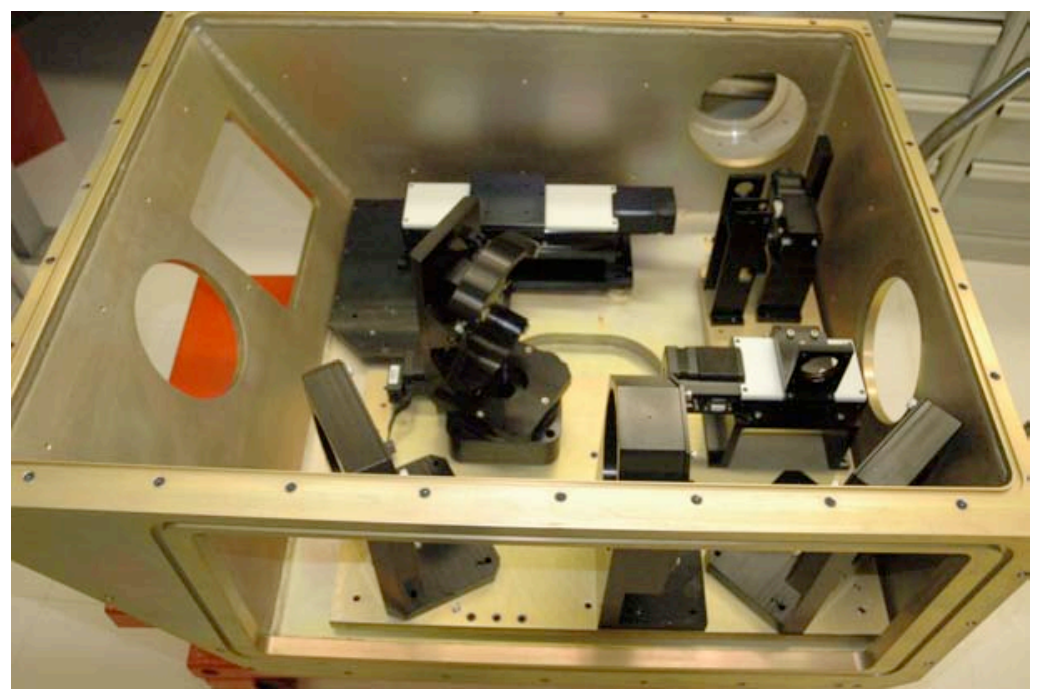

Fig. 7. The interior of the Optics Box at present. The stage and optics holders have been installed and work on the control software has been begum. All of the optical components have already been either acquired or manufactured. However, they have not yet been installed in the box. 
CASIMIR will use the fully reflective tertiary mirror on SOFIA's telescope. As a result, none of the observatory's guiding cameras will be able to image the telescope's focal plane. Therefore, we have included an optical boresight camera, inside the Optics Box, for alignment and beamfinding. The boresight can also be used as a pupil imager by moving a biconcave lens into the optical path. The camera has a $6^{\prime} \times 6^{\prime}$ field of view and uses a $1024 \times 1024$ pixel, optical wavelength CCD. The rotating mirror also selects this camera.

Stepper motors are used to move all the optical components. All of these motors are mounted inside the Optics Box and are controlled remotely via software. There are only electronic feedthroughs mounted in the sides of the box, without any mechanical motions through the pressure boundary. Physical access to the Optics Box will not be required at any time during the flight.

\subsection{Cryostats}

The cryostats are of conventional design with $\mathrm{LN}_{2}$ and LHe reservoirs. For frequencies below $1 \mathrm{THz}$, the mixers will operate at $\sim 4 \mathrm{~K}$. At higher frequencies, we will pump on the LHe reservoir to operate the receivers at $\sim 2.5 \mathrm{~K}$.

There will be two cryostats per flight and up to two frequency bands in each cryostat, so there will be up to four bands available per flight. Observations can be made with only one band at a time. Any one of the four bands can be selected at anytime during the flight. This selection is made by software alone, and does not require caging of the telescope, any mechanical adjustment or physical access to the instrument.

As shown in Figures 8 and 9, all the components specific to an individual frequency band are integrated directly onto the cryostat, i. e., the LOs, IF systems, and relay optics. All systems mounted elsewhere on the instrument are common to all of the bands. Therefore, changing the selection of the four bands which are to be available on a given flight only requires swapping cryostats, which could easily be carried out between flights. Also, any upgrades and improvements to the bands could be accomplished completely independent of the rest of the instrument. This will allow continuous upgrades to the frequency bands, throughout the life of the instrument.

\subsection{FIR relay optics}

Figure 9 shows a schematic of the relay optics, which uses two off-axis elliptical mirrors to match the incoming telescope beam to the output beam of the mixer. Including the telescope, there are five mirrors at ambient temperature and one cryogenically cooled mirror, EM1, in the optical train. This includes the two off-axis elliptical mirrors, the rotating, beam selecting plane mirror in the Optics Box, see Figure 6, and the fully reflective tertiary. The window in the base of the cryostat is the only pressure boundary in the optical path from the telescope. Therefore, this window and a lens in the mixer assembly are the only transmissive elements in the entire optical train from the telescope to the receiver.

The relay optics for all bands are designed to have an edge taper of $10 \mathrm{~dB}$. This corresponds to an aperture efficiency of 0.71 . Initially SOFIA will use an oversize tertiary mirror, which would reduce the aperture efficiency to 0.64 , however, a smaller tertiary mirror may become available later, increasing this efficiency.

All bands will have a main beam efficiency of 0.77 . The beam size for $550 \mathrm{GHz}$, i. e., the largest, is 0.8 arcmin.

\section{CONCLUSIONS}

CASIMIR is a FIR/Submm, heterodyne spectrometer for SOFIA. It is well suited for the studies of the warm $(T \sim 100 \mathrm{~K})$ interstellar medium, particularly water, measuring many significant lines unobservable from the ground. Initially, the instrument will cover 500 to $1400 \mathrm{GHz}$. Eventually the frequency coverage may be extended up to $2 \mathrm{THz}$. CASIMIR will have unprecedented sensitivity in this frequency region, due to recent advancement in SIS mixer design.

There will be up to 4 channels available per flight of the observatory. Any one of these channels can be selected at any time during the flight.

All bands will have an IF bandwidth of $4 \mathrm{GHz}$. A FFT digital spectrometer will provide continuous coverage of this band with very high resolution, up to greater than $10^{6}$.

The instrument design is extremely modular and will be able to continuously incorporate new hardware and accommodate future improvements in mixer, LO and microwave spectrometer technologies, throughout the lifetime of the SOFIA observatory. 


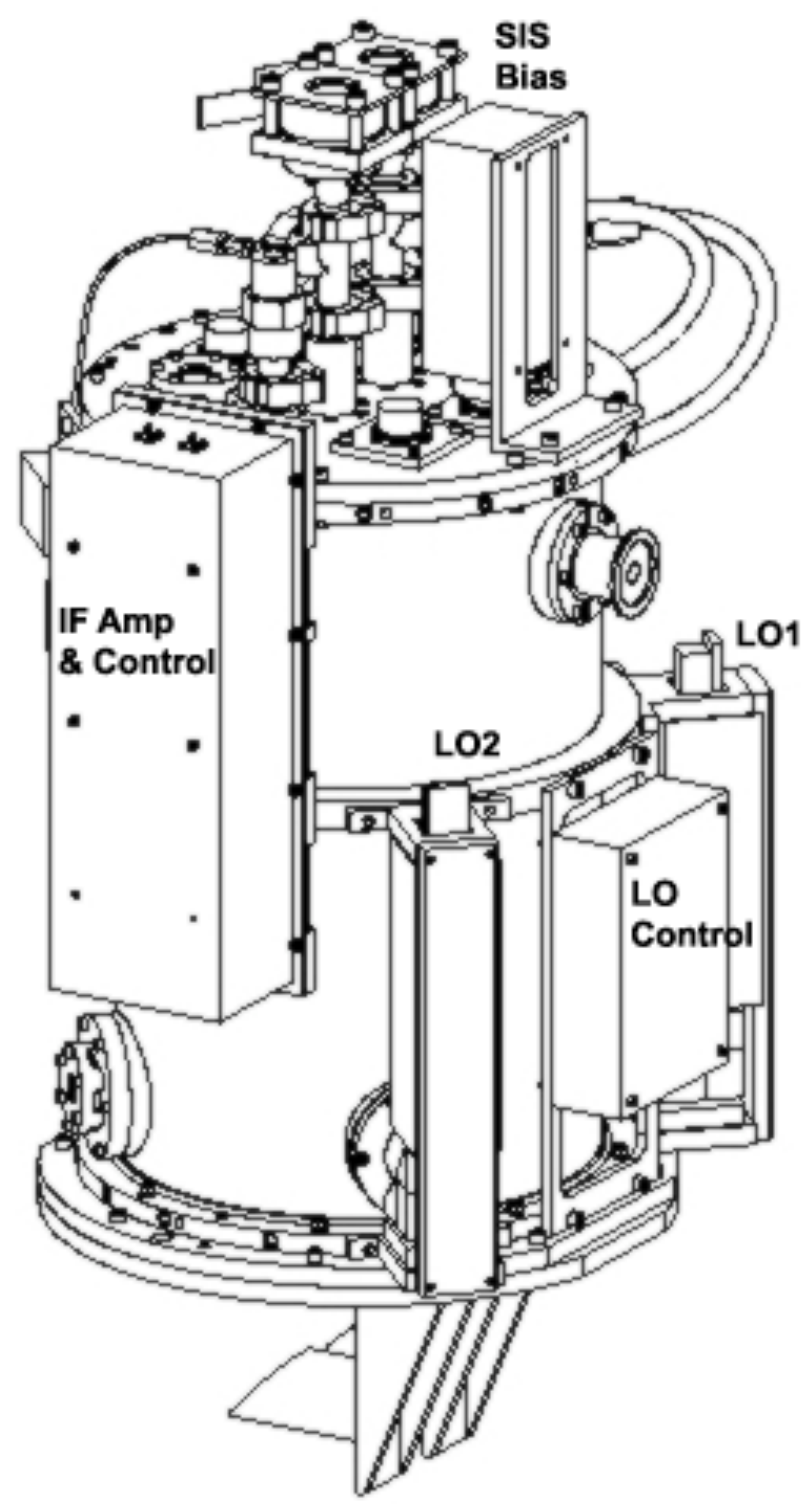

Fig. 8. The CASIMIR Cryostat. The cryostat contains 5 liters each of $\mathrm{LN}_{2}$ and $\mathrm{LHe}$ and has a $250 \mathrm{~mm}$ diameter cold-worksurface. This is the approximate, maximum, practical diameter for cryostats that can be used in the side-by-side configuration for SOFIA. It is $600 \mathrm{~mm}$ high and weighs $\sim 40 \mathrm{~kg}$. The LOs, IF system, receiver and LO bias electronics are mounted directly to the side of the cryostat. The electronics for the cryogenic amplifier bias and mixer electromagnet current are also mounted on the cryostat but are not shown in this view. The rather impressive array of plumbing fixtures on the top of the cryostat prevents the formation of ice plugs or the rupture of the cryogenic reservoirs. This design was required to obtain airworthiness certification of the cryostat by the Federal Aviation Administration. The two elliptical mirrors of the relay optics, mounted on the base of the cryostat, can be seen at the extreme bottom of the image. 

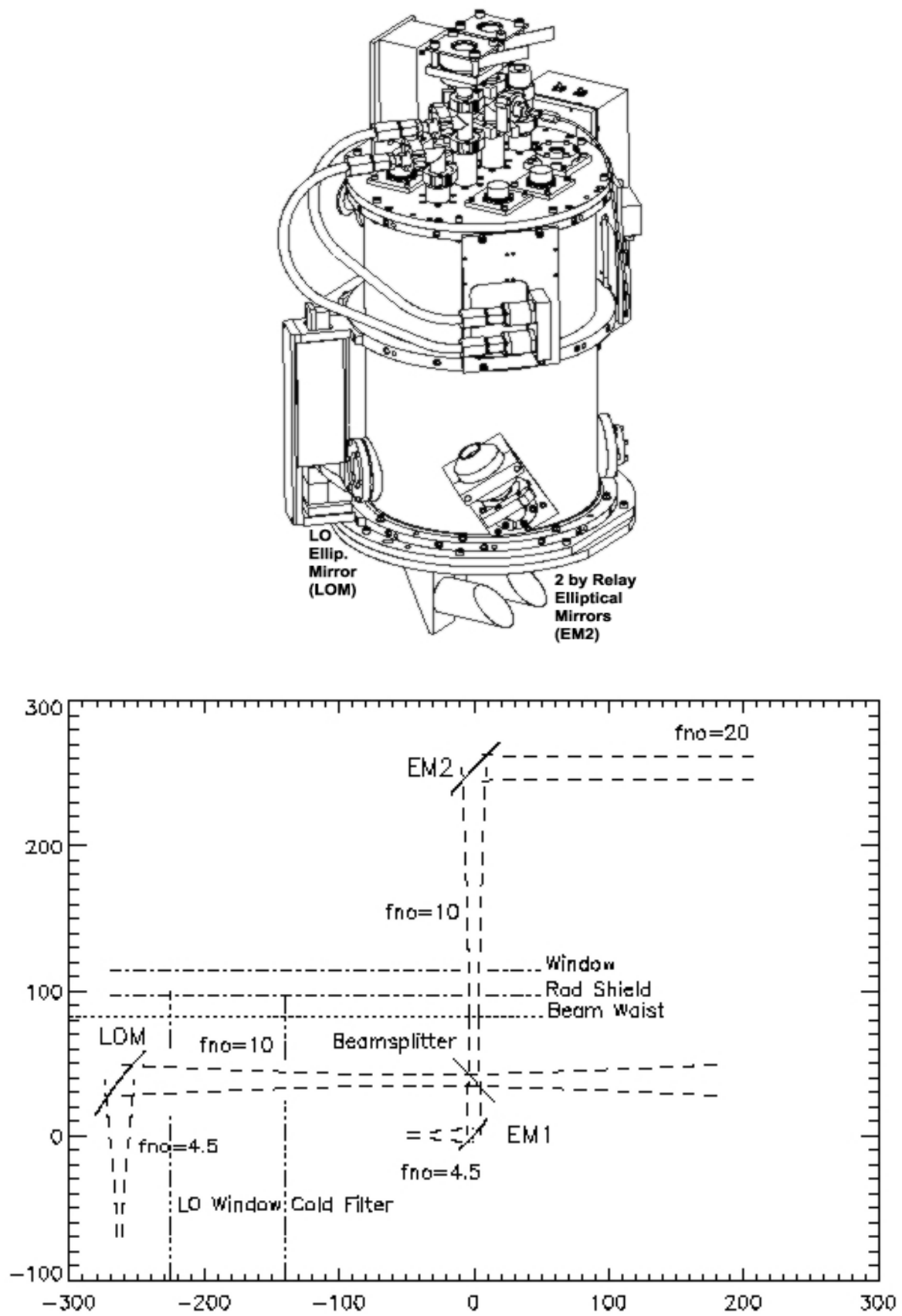

Fig. 9. CASIMIR Relay and LO Injection Optics. The top image shows the location of the elliptical mirrors mounted on the cryostat. The bottom part of the image shows a schematic of the optics. In the lower image, the up down orientation is reversed, compared to the cryostat, and the units on the scale are $\mathrm{mm}$, with the origin at the center of EM1, the elliptical mirror mounted on the cryostat cold-work surface. EM2 is the elliptical mirror mounted below the base of the cryostat, visible in the left image. EM2 is in the plane of the telescope beam, it converts the incoming, diverging $f / 20$ telescope beam into an intermediate $f / 10$ beam and reflects it through $90^{\circ}$, through a window in the base of the cryostat. LOM is the LO elliptical mirror, which matches the LO output beam to the incoming intermediate beam. EM1 converts the intermediate beam to a converging $\sim f / 4.5$ beam, which matches the output beam of the mixer. 


\section{ACKNOWLEDGEMENTS}

Various subsystems of the CASIMIR instrument have been or continue to be developed by a number of people at several institutions: H. G. Le Duc, Micro Devices Lab, JPL (mixer fabrication), N. R., M. R. Haas, NASA Ames (optics), the Kosma I/O Team, U. of Köln and S. W. Colgan, NASA Ames (software). The development of CASIMIR is supported by NASA/USRA SOFIA Instrument Development Fund.

\section{REFERENCES}

[1] Casey, S. C., "The SOFIA program: astronomers return to the stratosphere," Proc. SPIE 6267 (2006).

[2] Becklin, E. E, Tielens, A. G. G. M., and Callis, H. H. S., "Stratospheric Observatory for Infrared Astronomy (SOFIA)," Mod. Phys. Lett. A 21, 2551-2560 (2006).

[3] Neufeld, D. A., and Kaufan, M. J. "Radiative Cooling of Warm Molecular Gas," Ap. J. 418, 263 (1993).

[4] Vastel, C., Caselli, P., Ceccarelli, C., Phillips, T., Wiedner, C., Peng, R., Houde, M., and Dominik, C., "The distribution of ortho- $\mathrm{H}_{2} \mathrm{D}^{+}\left(1_{1,0}-1_{1,1}\right)$ in L1544: tracing the deuterization factory in prestellar cores," $A p . J$. 645 , 1198-1211 (2006).

[5] Harju, J., Haikala, L. K., Lehtinen, K., Juvela, M., Matilla, K., Miettinen, O., Dumke, M., Gusten, R., and Nyman, L.-A., "Detection of H2D" in a massive prestellar core in Orion B*," $A \& A$ 454, L55-58 (2006).

[6] Boreiko, R. T., and Betz, A. L., "A search for the rotational transitions $\mathrm{H}_{2} \mathrm{D}^{+}$at $1370 \mathrm{GHz}$ and $\mathrm{H}_{3} \mathrm{O}^{+}$at $985 \mathrm{GHz}$," Ap. J. (Letters) 405, L39-L42 (1993).

[7] Karpov, A., Miller, D., Rice, F., Stern, J.A., Bumble, B., LeDuc, H.G., \& Zmuidzinas, J., "Low Noise 1 THz-1.4 THz Mixers Using Nb/Al-AlN/NbTiN SIS Junctions," IEEE Trans. Applied Superconductivity 17, 343 (2007).

[8] Virginia Diodes Inc., 979 Second St. S.E., Suite 309, Charlottesville, VA 22902-6172, USA.

[9] Mehdi, I., Schlect, E., Chattopadhyay, G., and Siegel, P. H., "THz local oscillator sources: performance and capabilities," Proc. SPIE 4855, 435-446, (2003).

[10] Wadefalk, N., Mellberg, A., Angelov, I., Barsky, M. E., Bui, S., Choumas, E., Grundbacher, R. W., Kollberg, E. L., Lai, R., Rorsman, N., Starski, P., Stenarson, J., Streit, D. C., and Zirath, H., "Cryogenic wide-band ultra-low-noise IF amplifiers operating at ultra-low DC power," IEEE Trans. Microwave Theory Tech. 51, 1705-1711 (2003).

[11] CTT Inc., 3005 Democracy Way, Santa Clara, CA 95054, USA.

${ }^{[12]}$ Emrich, A., Krus, M., and Reisbeck, J., "FFT spectrometer for (sub)mm radiometer applications," Proc. 18th Int. Symp. Space Terahertz Tech., 140 (2007).

[13] Omnisys Instruments AB, Gruvgatan 8, 42130 Goteborg, Sweden, www.omnisys.se 